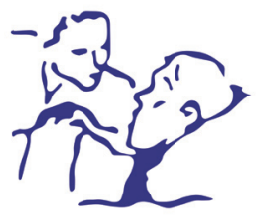

\title{
Organización paliativa durante la pandemia de la COVID-19 y propuestas para la adaptación de los servicios y programas de cuidados paliativos y de atención psicosocial ante la posibilidad de reactivación de la pandemia y época pos-COVID-19
}

\author{
Xavier Gómez-Batiste ${ }^{1,2}$, Juan P. Leiva ${ }^{3}$, Albert Tuca ${ }^{4}$, Elba Beas ${ }^{1,2}$, Rosa M. Montoliu ${ }^{1,2}$, \\ Rafael Gálvez ${ }^{5}$, Jordi Trelis ${ }^{6}$, Sebastià Santaeugenia ${ }^{7}$ y Jordi Amblàs ${ }^{1,8}$
}

${ }^{1}$ Cátedra de Cuidados Paliativos, Universitat de Vic/Central de Catalunya. Vic, Barcelona, España. ${ }^{2}$ Observatorio Qualy. Instituto Catalán de Oncología. Barcelona, España. ${ }^{3}$ Equipo de Soporte y Cuidados Paliativos, Hospital de Manacor, Mallorca, España. ${ }^{4}$ Unidad de Cuidados de Soporte y Paliativos en Cáncer, Servicio de Oncología Médica, Hospital Clínico de Barcelona. Cátedra de Cuidados Paliativos, Universidad de Barcelona-Hospital Clínic. Universidad de Barcelona, España. ${ }^{5}$ Unidad del Dolor y Equipo de Atención Psicosocial, Hospital Universitario Virgen de las Nieves. Granada. Profesor Titular Cuidados Paliativos UGR Granada, España. ${ }^{6}$ Cuidados Paliativos, Instituto Catalán de Oncología. Barcelona, España. 7Programa de Prevenció i Atenció a la Cronicitat de Catalunya (PPAC), Departament de Salut, Generalitat de Catalunya. Barcelona, España. ${ }^{8}$ Grup de Recerca en Cronicitat de la Catalunya Central (C3RG), Centre d'Estudis Sanitaris i Socials. Universitat de Vic/Central de Catalunya. Vic, Barcelona, España

Recibido el 1 de julio de 2020

Aceptado el 16 de octubre de 2020

PALABRAS CLAVE

Cuidados paliativos, intervención psicosocial, COVID-19, pandemia.

\begin{abstract}
Resumen
Introducción: La irrupción brusca e intensa de la COVID-19 en nuestra sociedad ha provocado un elevado sufrimiento en pacientes y familias, así como una elevada presión en profesionales y servicios. Sus peculiares características han hecho emerger la atención paliativa como uno de los ejes principales de su abordaje. Este hecho ha provocado una reestructuración de equipos y servicios de cuidados paliativos y atención psicosocial.

Objetivos: Describir la respuesta inicial de diversos equipos de cuidados paliativos y atención psicosocial ante la pandemia por la COVID-19, así como elaborar una serie de recomendaciones ante la posibilidad de una nueva reactivación de la pandemia.

Material y métodos: Tras una revisión narrativa de la literatura se elaboró un cuestionario con los elementos organizativos más destacados. Este cuestionario, se envió a diversos expertos y referentes en cuidados paliativos en España. En una segunda fase, y tras el análisis de los resultados del cuestionario, se elaboraron una serie de recomendaciones.
\end{abstract}

\footnotetext{
*Autor para correspondencia:

Xavier Gómez-Batiste

Cátedra y Máster de Cuidados Paliativos, Facultad de Medicina, Universitat de Vic/Central Catalunya, UVIC/UCC. C/ Marti Pol, N. ${ }^{\circ} 1.08500$, Vic, Barcelona

Correo electrónico: xgomez@iconcologia.net
}

DOI: $10.20986 /$ medpal.2020.1184/2020

1134-248X/@ 2020 Sociedad Española de Cuidados Paliativos. Publicado por Inspira Network. Todos los derechos reservados. 


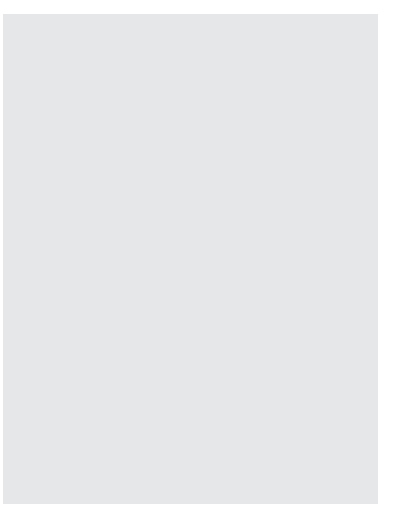

Resultados: Los elementos destacados de la revisión de la literatura fueron: 1) preparación y protección de equipos; 2 ) presencia de los cuidados paliativos en el sistema; 3 ) intervención en pacientes agudos; 4) desarrollo de planes asistenciales; 5) provisión de medicación esencial; 6) voluntariado; 7) docencia, y 8) participación de la sociedad. Respondieron 26 de los 31 expertos. Los resultados muestran una respuesta organizativa variable, desde implicación y liderazgo hasta reducción de estructuras, de acuerdo con su posición previa en el sistema. Dentro de las propuestas para afrontar una nueva ola de la pandemia se encuentran: 1) evaluación del estado de los equipos; 2) valorar el grado de adaptación y respuesta a la crisis; 3) conocer las nuevas necesidades; 4) promover una intervención proactiva; y 5) reprogramar los actuales planes de cuidados paliativos.

Conclusiones: El presente trabajo muestra la respuesta inicial global de los equipos paliativos ante la pandemia por la COVID-19 y propone una serie de recomendaciones sobre las que plantear intervenciones futuras que respondan a la futura realidad pos-COVID-19.

\section{KEYWORDS}

Palliative care, psychosocial care, COVID-19, pandemic.

\begin{abstract}
Introduction: The sudden and intense COVID-19 outbreak has caused in our society a lot of suffering in patients and families alike, and has also put high pressure on professionals and services. Its peculiar characteristics have led to the emergence of palliative care as one of the main axes for its approach. This fact has led to a restructuring of palliative care and psychosocial care teams and services.
\end{abstract}

Aims: To describe the initial response of various palliative care and psychosocial care teams to the COVID-19 pandemic, as well as to draw up a series of recommendations regarding the possibility of a new reactivation of the pandemic.

Material and methods: After a narrative review of the literature, a questionnaire was prepared with the most outstanding organizational elements. This questionnaire was sent to various experts and opinion leaders in palliative care in Spain. In a second phase, and after analyzing the results of the questionnaire, a series of recommendations were issued.

Results: The highlighted elements of the literature review were: 1 ) equipment preparation and protection; 2 ) the presence of palliative care in the health system; 3 ) intervention in acute patients; 4) development of care plans; 5) provision of essential medication; 6) volunteering; 7) teaching; and 8) civil society participation. A total of 26 out of 31 experts responded. The results show a variable organizational response, from involvement and leadership to reduction of structures, according to the position previously held in the system. Among the proposals to face a new wave of the pandemic are: 1 ) evaluating the state of equipments; 2 ) assessing the degree of adaptation and response to the crisis; 3) satisfying new needs; 4) promoting proactive intervention; and 5) reprograming current palliative care plans.

Conclusions: This work shows the initial global response of palliative teams to the COVID-19 pandemic, and issues a series of recommendations on which future interventions to respond to post-COVID-19 reality should be based.

Gómez-Batiste X, Leiva JP, Tuca A, Beas E, Montoliu RM, Gálvez R, et al. Organización paliativa durante la pandemia de la COVID-19 y propuestas para la adaptación de los servicios y programas de cuidados paliativos y de atención psicosocial ante la posibilidad de reactivación de la pandemia y época pos-COVID-19. Med Paliat. 2020;27(3):242-254

\section{INTRODUCCIÓN}

La pandemia por la COVID-19 ha irrumpido en la sociedad bruscamente, interfiriendo en la actividad de todos los programas de cuidados paliativos y en sus equipos. Este hecho ha provocado cambios sustanciales en la atención paliativa: cambios en las necesidades de los pacientes, dificultades de acceso presencial, adaptación urgente de las actividades y aparición de nuevas fórmulas de intervención, como la atención telemática.

Su inicio repentino, así como las características de una pandemia (capacidad de contagio, severidad, morbimortalidad, impacto social, sanitario y económico y efecto global, entre otros), provocan que estos cambios se desarrollen a corto, medio y largo plazo. 
Desde un punto de vista organizativo, esta situación ha provocado que los Equipos y Servicios de Cuidados Paliativos (SCP), así como los Equipos de Atención Psicosocial (EAPS), hayan tenido que responder inicialmente a la fase aguda de la pandemia, en función de su estructura, posición en el sistema, capacidad de liderazgo y presencia asistencial. Por otra parte, se deben realizar planes específicos para prepararse ante nuevas reactivaciones de esta pandemia.

Como centro dedicado al asesoramiento en la organización en programas de Cuidados Paliativos, y referente en la docencia en este ámbito, la Cátedra de Atención y Cuidados Paliativos ICO/UVIC ha realizado diversas iniciativas para colaborar en la lucha contra la pandemia por la COVID-19. Entre estas destacan la elaboración de documentos de soporte a la toma de decisiones en residencias ${ }^{1,2}$ o unidades de cuidados intensivos $(\mathrm{UCl})^{3}$. En el contexto del programa para la atención integral de personas con enfermedades avanzadas de la Obra Social de la Caixa ${ }^{4}$, y con el rol de dirección científica, se creó un call center de soporte psicológico a profesionales ${ }^{5}$, un directorio de recursos de conocimiento actualizado en aspectos generales y psicosociales ${ }^{6}$, así como la reordenación y soporte de los equipos de atención psicosocial y de programas de soledad al final de la vida. Se realizaron múltiples intervenciones públicas en medios audiovisuales y escritos ${ }^{7,8}$. Asimismo, se modificó el formato presencial de las actividades docentes habituales a versión online.

Siguiendo esta misma línea, se decidió analizar la respuesta organizativa de diversos equipos e instituciones en cuidados paliativos ante la pandemia. Así pues, el objetivo del presente trabajo es describir las respuestas de los SCP y EAPS en la fase aguda de la pandemia y proponer una serie de recomendaciones para su adaptación a nuevas reagudizaciones de la pandemia por la COVID-19.

\section{METODOLOGÍA}

Para dar respuesta a los objetivos planteados se organizó el trabajo en tres fases. Una primera fase consistente en una revisión narrativa de la literatura. Tras la extracción de los elementos organizativos principales, se elaboró un cuestionario específico y se administró a expertos de cuidados paliativos de diversas localizaciones de España para describir la respuesta organizativa en sus centros. Por último, se consensuaron las aportaciones de este panel de expertos ante escenarios futuros. Estos expertos fueron seleccionados por ubicación geográfica y posición en el sistema.

\section{Revisión de la literatura}

Se realizó una revisión narrativa de la literatura utilizando las palabras clave: "palliative care AND covid"; "palliative care AND pandemic"; "palliative care AND humanitarian crisis".

Los modelos encontrados en la literatura revisada de respuesta a la pandemia que utilizamos han sido los de la revisión de respuestas a pandemias publicadas anteriormente $e^{9-13}$, hasta algunos de respuesta CP a la pandemia COVID-19 de carácter general ${ }^{14-21} \mathrm{o}$ específicos para alguna de las dimensiones $^{22-25}$. La otra fuente de información utilizada fue el
Directorio de recursos de la Obra Social de La Caixa, que recogió la información más relevante durante los meses de marzo a junio de $2020^{26}$.

\section{Cuestionario y entrevista a expertos}

Tras la revisión de la literatura se obtuvieron diversos elementos organizativos de respuesta a la pandemia. Con ello se elaboró una encuesta autoadministrada y semiestructurada con dos tablas: en la Tabla I se describe la respuesta a la fase de crisis aguda COVID-19, y en la Tabla II se especifican las recomendaciones para la respuesta a la fase posterior COVID-19. Se añadió un análisis de debilidades, amenazas, fortalezas y oportunidades (DAFO) (Tablas III y IV) y un apartado de respuestas abiertas (Tabla V). Las áreas exploradas fueron: 1) preparación y protección de equipos; 2 ) presencia de los cuidados paliativos en el sistema; 3 ) intervención en pacientes agudos; 4) desarrollo de planes asistenciales; 5) provisión de medicación esencial; 6) voluntariado; 7) docencia, y 8) participación de la sociedad.

\section{Análisis de la encuesta de opinión y percepciones de los expertos}

Para llevar a cabo el análisis cualitativo de las respuestas emitidas se han seguido los siguientes procedimientos: 1 ) fase de descubrimiento (organización y lectura detenida de los datos identificando temas, intuiciones preanalíticas y posibles interpretaciones); 2) fase de análisis (segmentación de textos, codificación y agrupación en categorías); y 3) fase de relativización (verificación y contraste de los hallazgos). Para ilustrar las categorías se han seleccionado citas textuales. La codificación de las entrevistas autoadministradas se ha triangulado entre dos de los miembros del equipo investigador buscando de este modo la fiabilidad del proceso.

\section{RESULTADOS}

\section{Revisión de la literatura}

Los resultados globales de la revisión rápida incluyen un total de 61 artículos, agrupados por temas (organización, aspectos asistenciales directos, psicosociales, formativos), desde febrero hasta abril de 2020. Adicionalmente, se ha creado un repositorio de información sobre atención paliativa y psicosocial COVID-19 accesible en https: / /obrasociallacaixa.org/es/atencion-integral-enfermedades-avanzadas-la-caixa (el listado de documentos revisados aparece en forma de anexo en la página web de la revista Medicina Paliativa).

\section{Cuestionario y entrevista a expertos}

Obtuvimos un total de $26 / 31(80,6 \%)$ respuestas, (18/23 expertos y $8 / 8$ autores). Describimos los resultados en las Tablas I, II y III. 
Tabla I. Respuestas iniciales de servicios de cuidados paliativos y equipos de atención psicosocial a la crisis por la COVID-19.

\begin{tabular}{|c|c|c|}
\hline Elementos & Objetivos & Actividad / Tipos de respuestas realizadas \\
\hline $\begin{array}{l}\text { Evaluación del } \\
\text { estado equipo }\end{array}$ & $\begin{array}{l}\text { - Evaluar y cuidar: } \\
\text { estado físico, } \\
\text { emocional, laboral, } \\
\text { del equipo } \\
\text { - Protección y apoyo } \\
\text { - Valorar recursos } \\
\text { disponibles }\end{array}$ & $\begin{array}{l}\text { - Valorar estado y capacidad de respuesta equipo y los profesionales con mayor } \\
\text { riesgo } \\
\text { - Asegurar la protección ante la COVID-19 (equipos de protección individual, } \\
\text { mascarillas, medidas...) } \\
\text { - Valorar situación emocional y estrategias de soporte mutuo } \\
\text { - Valoración de las características y situación del núcleo familiar de los miembros } \\
\text { del equipo } \\
\text { - Privacidad paciente, familia y equipo } \\
\text { - Reforzar autocuidado equipo } \\
\text { - Favorecer el teletrabajo (adecuación formativa y tecnológica) de los profesionales } \\
\text { para su protección y disminución de la intensidad de exposición al virus } \\
\text { - Mantener las sesiones de supervisión del equipo vía telemática y con su psicólogo } \\
\text { habitual }\end{array}$ \\
\hline $\begin{array}{l}\text { Presencia en } \\
\text { sistema y } \\
\text { respuestas }\end{array}$ & $\begin{array}{l}\text { - Atención paliativa } \\
\text { y psicosocial como } \\
\text { uno de los } \\
\text { elementos de } \\
\text { respuesta a } \\
\text { COVID-19 y } \\
\text { necesidades }\end{array}$ & $\begin{array}{l}\text { 1. Adaptación a normativas generales: protección, limitación acceso directo: } \\
\text { - Estrategias de prevención de la propagación y de las cadenas de contagio de la } \\
\text { COVID-19 en los pacientes atendidos por SCP y EAPS, en el contexto de las } \\
\text { directrices generales del hospital e instituciones sanitarias } \\
\text { - Medidas destinadas a evitar la frecuentación al hospital y centros sanitarios } \\
\text { - Reducir la movilidad de los pacientes y sus familias } \\
\text { - Atención virtual como alternativa a la visita presencial (visita telefónica o } \\
\text { videollamada) } \\
\text { - Promoción de confinamiento domiciliario } \\
\text { 2. Cambios organizativos con } 4 \text { objetivos: } \\
\text { - Mantener en lo posible la atención paliativa a las personas atendidas por los SCP } \\
\text { y EAPS } \\
\text { - Establecer una respuesta rápida a las necesidades de los pacientes críticos de la } \\
\text { COVID-19 y sus familias } \\
\text { - Asegurar las medidas de contención de la cadena de contagios SARS-CoV } 2 \text { para } \\
\text { proteger a los pacientes, sus familias, el personal sanitario y la población } \\
\text { general } \\
\text { - Contribuir en el conocimiento sobre la toma de decisiones basada en la } \\
\text { fragilidad de las personas y su influencia en las opciones de control de la } \\
\text { infección por la COVID } 19 \text { y las decisiones compartidas con el paciente, en un } \\
\text { contexto de incertidumbre y de recursos limitados } \\
\text { 3. Metodología: } \\
\text { - Planes de atención diseñados en contacto con planificadores, gestores, y líderes } \\
\text { sanitarios y sociales } \\
\text { - Propuestas fórmulas realistas de intervención } \\
\text { - Cambios en criterios y fórmulas de intervención: evaluación exprés, consultas } \\
\text { exprés }\end{array}$ \\
\hline $\begin{array}{l}\text { Aparición de } \\
\text { nuevas } \\
\text { necesidades y } \\
\text { demandas }\end{array}$ & $\begin{array}{l}\text { - Fase aguda: } \\
\text { aparición brusca de } \\
\text { necesidades } \\
\text { cuantitativas y } \\
\text { cualitativas } \\
\text { - Identificación de } \\
\text { servicios más } \\
\text { afectados }\end{array}$ & $\begin{array}{l}\text { - Pacientes agudos con sintomatología, impacto emocional, dilemas éticos y } \\
\text { necesidades complejas de aparición aguda } \\
\text { - Familias con impacto aumentado por aparición súbita, mortalidad, incertidumbre } \\
\text { e imposibilidad de presencia } \\
\text { - Elevada mortalidad } \\
\text { - Incertidumbre } \\
\text { - Objetivo curativo y respuesta aguda predominantes } \\
\text { - Servicios de agudos, urgencias, UCI colapsados } \\
\text { - Profesionales en fase de respuesta aguda } \\
\text { - Dilemas de asignación de recursos centrados en la atención más intensiva de } \\
\text { quienes tuvieran mayor probabilidad de recuperación } \\
\text { - Protección y contagio }\end{array}$ \\
\hline
\end{tabular}


Tabla I (Cont.). Respuestas iniciales de servicios de cuidados paliativos y equipos de atención psicosocial a la crisis por la COVID-19.

\begin{tabular}{|c|c|c|}
\hline Elementos & Objetivos & Actividad / Tipos de respuestas realizadas \\
\hline $\begin{array}{l}\text { Plan de } \\
\text { adaptación } \\
\text { asistencial }\end{array}$ & $\begin{array}{l}\text { Revisar plan de } \\
\text { trabajo } \\
\text { asistencial y } \\
\text { explorar nuevas } \\
\text { formas }\end{array}$ & 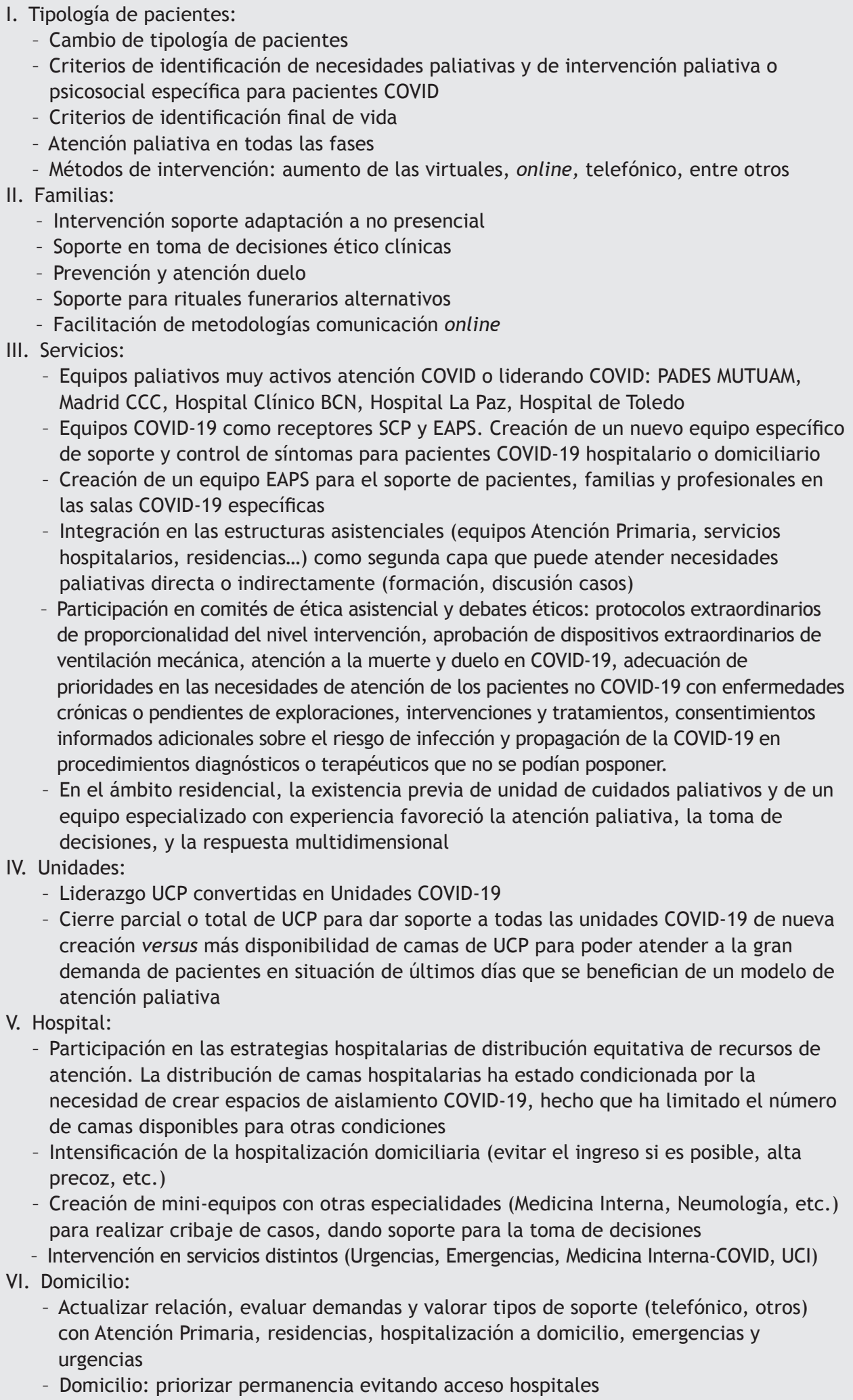 \\
\hline
\end{tabular}


Tabla I (Cont.). Respuestas iniciales de servicios de cuidados paliativos y equipos de atención psicosocial a la crisis por la COVID-19.

\begin{tabular}{|c|c|c|}
\hline Elementos & Objetivos & Actividad / Tipos de respuestas realizadas \\
\hline & $\begin{array}{l}\text { - Evaluar } \\
\text { necesidades y } \\
\text { explorar } \\
\text { nuevas } \\
\text { fórmulas de } \\
\text { soporte }\end{array}$ & $\begin{array}{l}\text { - Soporte a profesionales para atención directa pacientes: control síntomas, } \\
\text { comunicación, sedación, “PDA rápida” } \\
\text { - Decisiones ético clínicas, criterios y niveles de intervención } \\
\text { - Soporte emocional para adaptación } \\
\text { - Creación de consultas monográficas de atención bio-psico-social para profesionales con } \\
\text { síntomas COVID-19 } \\
\text { - Call Center } \\
\text { - Asistencia telemática o presencial } \\
\text { - Protocolos y foros de discusión: control de síntomas, soporte emocional, información, } \\
\text { toma decisiones, PDA, criterios de triaje, sedación, atención familia } \\
\text { - Evaluación de necesidades formativas }\end{array}$ \\
\hline Medicación & $\begin{array}{l}\text { - Asegurar } \\
\text { provisión }\end{array}$ & $\begin{array}{l}\text { - Elaboración de kits medicación control de síntomas } \\
\text { - Elaboración de kits medicación sedación }\end{array}$ \\
\hline Voluntariado & $\begin{array}{l}\text { - Revisar tipo de } \\
\text { actuación }\end{array}$ & $\begin{array}{l}\text { - Revisar intervenciones ante la imposibilidad de intervención directa } \\
\text { - Explorar nuevas fórmulas de soporte } \\
\text { - Voluntariado virtual mediante videoconferencia, soporte familiares }\end{array}$ \\
\hline $\begin{array}{l}\text { Docencia } \\
\text { Conocimiento }\end{array}$ & $\begin{array}{l}\text { - Nuevos } \\
\text { contenidos } \\
\text { docentes y de } \\
\text { educación y } \\
\text { soporte } \\
\text { - Directorio de } \\
\text { recursos }\end{array}$ & $\begin{array}{l}\text { - Protocolos: control de síntomas, comunicación COVID-19, atención familia, sedación, } \\
\text { últimos días, duelo... } \\
\text { - Nuevos formatos docentes (webinars, tutoriales, “cápsulas educativas”, etc.) } \\
\text { - Tutoriales del uso de algunos dispositivos; por ejemplo, se han hecho sobre el uso de las } \\
\text { bombas de infusión continua } \\
\text { - Formación rápida directa u online } \\
\text { - Infografías } \\
\text { - Nuevas dianas de formación: residencias, UCI, urgencias, centros de discapacidad }\end{array}$ \\
\hline $\begin{array}{l}\text { Involucrar } \\
\text { otros servicios } \\
\text { y programas }\end{array}$ & $\begin{array}{l}\text { - Exploración } \\
\text { alianzas con } \\
\text { otros servicios: } \\
\text { UCI, } \\
\text { emergencias, } \\
\text { residencias, } \\
\text { otros programas } \\
\text { Obra Social } \\
\text { Caixa (soledad, } \\
\text { pobreza, } \\
\text { inequidad, } \\
\text { etc.), } \\
\text { comunidades } \\
\text { cuidadoras }\end{array}$ & $\begin{array}{l}\text { - Contacto formal con los diferentes servicios y programas } \\
\text { - Modelos de intervención distintos } \\
\text { - Comunidades cuidadoras }\end{array}$ \\
\hline $\begin{array}{l}\text { Involucrar } \\
\text { sociedad }\end{array}$ & $\begin{array}{l}\text { - Explorar } \\
\text { necesidades y } \\
\text { aportar } \\
\text { propuestas }\end{array}$ & $\begin{array}{l}\text { - Contacto con líderes comunidad } \\
\text { - Medios / Prensa / Educación sociedad } \\
\text { - Información / Recomendaciones de autocuidado } \\
\text { - Recomendaciones para promover el cuidado de personas con alto riesgo y fragilidad }\end{array}$ \\
\hline
\end{tabular}

\section{Respuesta inicial del sistema salud ante irrupción de la pandemia por la COVID-19}

Ante la rapidez de instauración, la rapidez de extensión y contagio, la virulencia y agresividad de la infección, la respuesta inicial lógica ha sido la combinación de accesibilidad y ampliación de servicios urgencias, críticos y unidades de cuidados intensivos $(\mathrm{UCl})$, con adaptación urgente de servicios hospitalarios hacia las distintas formas de gravedad de la COVID-19, alternativas a hospitalización convencional (creación hospitales en naves y hoteles) de sustitución, asociadas a políticas de pre- vención (confinamiento, distribución de equipos de protección individual [EPI], distancia social, entre otros). No se priorizó la atención paliativa. No se desarrolló la atención comunitaria y primaria debido a la enorme presión de la respuesta aguda y las dificultades de los equipos convencionales de CP y los EAP para una reacción rápida. Los equipos estaban afectados por la propia pandemia, en cuarentena, con limitaciones en la intervención directa por las restricciones sociales, así como la asignación de nuevas tareas asistenciales. Así pues, las respuestas de los SCP y EAP han sido variables, en parte por la variabilidad de su posición prexistente en el sistema. 
Tabla II. Pasos para la respuesta pos-COVID-19.

\begin{tabular}{|c|c|c|}
\hline Paso & Objetivo & Actividad \\
\hline $\begin{array}{l}\text { 1. Evaluación estado } \\
\text { equipo }\end{array}$ & $\begin{array}{l}\text { Conocer estado físico, } \\
\text { emocional y laboral, pos-OVID }\end{array}$ & $\begin{array}{l}\text { - Autoevaluación } \\
\text { - DAFO: fortalezas, debilidades y requisitos para adaptarse a } \\
\text { COVID y pos-COVID }\end{array}$ \\
\hline $\begin{array}{l}\text { 2. Evaluación de } \\
\text { respuesta a COVID }\end{array}$ & $\begin{array}{l}\text { Valorar grado de adaptación y } \\
\text { respuestas a crisis }\end{array}$ & $\begin{array}{l}\text { - Reflexión sobre actividades, respuesta, dificultades en la crisis } \\
\text { - Elaboración de propuestas de respuesta a nuevas crisis y } \\
\text { pandemias }\end{array}$ \\
\hline $\begin{array}{l}\text { 3. Mapeo actualizado } \\
\text { de necesidades y } \\
\text { respuestas }\end{array}$ & $\begin{array}{l}\text { Conocer estado de necesidades, } \\
\text { los servicios, equipos receptores } \\
\text { previos y nuevos }\end{array}$ & $\begin{array}{l}\text { - Estimar cuantitativa y cualitativamente nuevas necesidades } \\
\text { - Encuesta semiestructurada a los actores clave } \\
\text { - Servicios, equipos: necesidades y demandas } \\
\text { - Especial atención a escenarios más afectados: } \\
\text { - Residencias: replantear el modelo de atención actual, con } \\
\text { recursos, formación y protocolización, insertándola atención } \\
\text { paliativa (identificación y atención), la planificación de } \\
\text { decisiones anticipadas, la gestión de caso) y la relación con } \\
\text { los equipos de soporte especializados } \\
\text { - Atención Primaria } \\
\text { - Nuevas unidades } \\
\text { - Emergencias, urgencias, UCI }\end{array}$ \\
\hline $\begin{array}{l}\text { 4. Contacto formal } \\
\text { con grupos de } \\
\text { interés }\end{array}$ & $\begin{array}{l}\text { - Identificar grupos de interés } \\
\text { para comunicación y perfilar } \\
\text { proyectos de colaboración } \\
\text { - Conocer sus necesidades y } \\
\text { demandas }\end{array}$ & $\begin{array}{l}\text { - Encuesta semiestructurada a grupos de interés, servicios, } \\
\text { equipos: ¿cómo podemos participar y generar colaboración } \\
\text { activa? } \\
\text { - Situación proactiva, identificándose con los actores que llevan } \\
\text { pacientes COVID-19 }\end{array}$ \\
\hline $\begin{array}{l}\text { 5. Plan de } \\
\text { adaptación } \\
\text { asistencial }\end{array}$ & $\begin{array}{l}\text { Revisar plan de trabajo } \\
\text { asistencial y explorar nuevas } \\
\text { formas }\end{array}$ & $\begin{array}{l}\text { - Reprogramación plan trabajo y estrategia a medio plazo, } \\
\text { incluyendo: } \\
\text { a Mantener y actualizar metodológicamente las actividades } \\
\text { previas (por ejemplo, soporte telemático, soporte telefónico, } \\
\text { etc.), incorporando lo experimentado / aprendido } \\
\text { b Identificar nuevas áreas de necesidades en las que el } \\
\text { COVID-19 ha generado: } \\
\text { - Impacto en familiares y duelo } \\
\text { - Impacto en profesionales } \\
\text { - Cronicidad + CovID-19 } \\
\text { - Necesidades sociales (soledad, pobreza, impacto social) y } \\
\text { programar respuesta paliativa, psicosocial y espiritual } \\
\text { especializada } \\
\text { c Identificar nuevos pacientes, actividades y métodos de } \\
\text { intervención } \\
\text { d Nuevos servicios receptores (residencias, servicios de agudos, } \\
\text { urgencias, etc.) } \\
\text { e Reorganizar voluntariado, incorporando nuevas fórmulas de } \\
\text { intervención, nuevas tipologías de voluntarios } \\
\text { f Identificar nuevos grupos de interés y alianzas: EAPS, } \\
\text { comunidades cuidadoras } \\
\text { g Preparar respuesta a nuevas crisis, incorporando lo aprendido }\end{array}$ \\
\hline $\begin{array}{l}\text { 6. Soporte } \\
\text { profesionales y } \\
\text { equipos }\end{array}$ & $\begin{array}{l}\text { Evaluar necesidades y explorar } \\
\text { nuevas fórmulas }\end{array}$ & $\begin{array}{l}\text { - Plan de trabajo soporte impacto en profesionales } \\
\text { - Evaluar necesidades, demandas de soporte, ayuda a toma } \\
\text { decisiones } \\
\text { - Grupos balint }\end{array}$ \\
\hline $\begin{array}{l}\text { 7. Docencia, } \\
\text { recomendaciones }\end{array}$ & $\begin{array}{l}\text { Nuevos contenidos docentes y } \\
\text { de educación y soporte }\end{array}$ & $\begin{array}{l}\text { - Plan docente incorporando nuevas necesidades } \\
\text { - Nuevos targets: de los servicios afectados (atención primaria, } \\
\text { residencias, agudos) } \\
\text { - Nuevos contenidos: COVID-19, aspectos éticos } \\
\text { - Fórmulas docentes telemáticas }\end{array}$ \\
\hline
\end{tabular}


Tabla II (Cont.). Pasos para la respuesta pos-COVID-19.

\begin{tabular}{|c|c|c|}
\hline Paso & Objetivo & Actividad \\
\hline $\begin{array}{l}\text { 8. Involucrar otros } \\
\text { servicios y } \\
\text { programas }\end{array}$ & $\begin{array}{l}\text { Explorar alianzas: otros } \\
\text { programas La Caixa (soledad, } \\
\text { etc.), comunidades cuidadoras }\end{array}$ & $\begin{array}{l}\text { - Contacto formal con los diferentes programas de comunidades } \\
\text { cuidadoras } \\
\text { - Involucrar con la óptica de la salud a otros colectivos } \\
\text { profesionales que también velan por la salud de sus colectivos y } \\
\text { ello les inspire a promover salud en la sociedad }\end{array}$ \\
\hline $\begin{array}{l}\text { 9. Involucrar } \\
\text { sociedad }\end{array}$ & $\begin{array}{l}\text { Explorar necesidades y aportar } \\
\text { propuestas }\end{array}$ & $\begin{array}{l}\text { - Identificar líderes de grupos vulnerables } \\
\text { - Identificar actores de respuestas ante la COVID-19 } \\
\text { - Contacto con líderes comunidad: evaluar nuevas necesidades y } \\
\text { demandas sociales: duelo, soledad, pobreza, impacto } \\
\text { - Contactar con programas existentes de comunidades cuidadoras } \\
\text { - Proponer nuevas fórmulas de respuesta a necesidades }\end{array}$ \\
\hline $\begin{array}{l}\text { 10. Insertarse en } \\
\text { sistema }\end{array}$ & $\begin{array}{l}\text { - Atención paliativa psicosocial } \\
\text { como uno de los elementos de } \\
\text { respuesta a la pandemia por } \\
\text { COVID-19 y necesidades } \\
\text { - Atención y soporte a duelo, } \\
\text { profesionales y vulnerables en } \\
\text { la cartera de servicios }\end{array}$ & $\begin{array}{l}\text { - Contacto con planificadores sanitarios y sociales } \\
\text { - Reformular el compromiso y posición en el sistema } \\
\text { - Atención paliativa y psicosocial como derechos y uno de los ejes } \\
\text { de la respuesta } \\
\text { - Esfuerzo educacional profesional y social sobre los elementos } \\
\text { clave de la atención paliativa } \\
\text { - Debate ético sobre el abordaje paliativo durante la pandemia } \\
\text { por la COVID-19 }\end{array}$ \\
\hline
\end{tabular}

Tabla III. Análisis DAFO de la respuesta de los equipos de cuidados paliativos en la pandemia por COVID-19 (I).

\begin{tabular}{ll}
\hline \multicolumn{1}{c}{ Debilidades / áreas de mejora } & \multicolumn{1}{c}{ Amenazas } \\
\hline - La presencia e influencia de los cuidados paliativos en la toma & - Protagonismo curativo y preventivo como respuesta \\
decisiones políticas sanitarias son débiles & prioritaria, especialmente en fases iniciales \\
- Escaso reconocimiento administrativo de las plazas & - Nuevo escenario en un contexto de predominio \\
específicas, o inexistencia de los cuidados paliativos como & hospitalario, carácter agudo, urgente y a veces de corta \\
especialidad, y su escasa presencia académica formal & duración del problema sanitario. Profesionales de CP o \\
- Estructura limitada de los servicios & los EAPS poco demandados \\
- Actitudes: & - Ocasionalmente, las indicaciones originales para el \\
- Estacionados en la "zona de confort" de pacientes & triaje y selección de pacientes candidatos para UCI no \\
terminales cáncer en nuestros servicios & contemplaban frecuentemente indicaciones de enfoque \\
- Poca flexibilidad & paliativo de control de síntomas, confort y alivio en los \\
- Ausencia de espíritu líder por parte de responsables de CP & no candidatos \\
- Poca formación en competencias de liderazgo & - Oferta de psicólogos ofertada por la administración, \\
- Ausencia de visión de las oportunidades mismas que la & colegio de psicólogos, colegio de médicos y voluntarios \\
pandemia puede generar & - La tarea asistencial de los SCP y EAPS la han realizado \\
- Victimismo, posición pasiva de queja & los profesionales de otras especialidades \\
- Formativa: nuevas necesidades y tipologías, nuevas & - Atención paliativa básica por servicios generalistas \\
fórmulas de trabajo que requieren formación & según posibilidades de respuesta y limitación en \\
- La mayoría de equipos de CP realizando trabajo telemático & recursos, de formación y protocolos \\
- Otros profesionales han asumido la intervención paliativa & - Indicios de utilización no protocolizada y de los \\
presencial & llamados "kits de sedación", como respuesta de equipos \\
- Escasa presencia y visibilidad social y sanitaria & sin formación específica y presión emocional y \\
& asistencial
\end{tabular}

Por otra parte, con un sistema de salud colapsado, los pacientes crónicos complejos y avanzados no-COVID, tradicionalmente atendidos por SCP y EAP, han sufrido las limitaciones de acceso al sistema convencional.

En el sistema de atención psicosocial y comunitario de voluntariado también han ocurrido hechos similares, con una primera fase de paralización y, posteriormente, el desarrollo de iniciativas espontáneas de soporte a pacientes y familiares y profesionales.

En la Tabla I se describen las respuestas específicas. Como se puede observar, estas han sido muy variables en función del estado del equipo, su capacidad de reacción y adaptación y su presencia previa en el sistema. Su capacidad de influencia en la respuesta y en relación con otros servicios ha variado desde el liderazgo a la marginalidad. 
Tabla IV. Análisis DAFO de la respuesta de los equipos de cuidados paliativos en la pandemia por COVID-19 (II).

\begin{tabular}{ll}
\multicolumn{1}{c}{ Fortalezas } & Oportunidades \\
\hline - Experiencia asistencial en atención integral de & - La crisis por COVID-19 ha permitido visualizar las situaciones de \\
personas con enfermedades avanzadas & necesidades, objetivo tradicional atención paliativa y psicosocial \\
- Experiencia en atención de pacientes complejos, & - Visualización social del duelo, de la necesidad de “morir bien”, \\
familias y equipos & del acompañamiento en la soledad y de la fatiga por compasión \\
- Experiencia en toma de decisiones ético clínicas, la & - Los CP pueden posicionarse como líder para respuestas potentes \\
planificación de decisiones anticipadas & e innovadoras \\
- Experiencia de trabajar bajo incertidumbre & - La pandemia puede activar la puesta en común de estrategias \\
- Diversidad de servicios y programas & integradas entre varios programas \\
- Reputación en la sociedad y sistema & - Nuevos modelos de organización y cooperación con otros equipos \\
- Posicionamiento social y sanitario con capacidad & o unidades \\
influencia en la respuesta a la crisis & - Nuevas áreas con necesidades paliativas: UCl, urgencias, \\
- La relación con la comunidad & unidades COVID, residencias \\
& - Necesidades de formación en atención paliativa, aspectos éticos \\
& y técnicas de comunicación \\
& - Sociedad vulnerable. Oportunidad para reflexionar sobre la \\
& necesidad de planificación de cuidados anticipados
\end{tabular}

Tabla V. Categorías emergentes de los comentarios de los expertos consultados.

Categorías emergentes
Participación de los equipos
de cuidados paliativos y
atención psicosocial e
integración en los recursos
sanitarios

- Experiencias semejantes salvo por variantes relacionadas con la organización de la atención según el territorio

- Importancia de la integración en las instituciones y referenciados en los diferentes recursos del sistema sanitario y social de la comunidad

- Conveniencia de ofrecer una atención integral a todas las personas con necesidades de atención paliativa y/o en situación de final de vida, independientemente del recurso en el que se encuentren

- En general, equipos alejados de la realidad sanitaria por decisiones jerárquicas y organizativas, en algún caso aislado también por decisión propia

- Baja participación y en algunos casos ha quedado incluso anulada y siendo sustituida por otras especialidades médicas que carecen de formación específica para asumir de forma exclusiva este tipo de atención integral psicosocial

- Destacan la competencia en valores junto con las técnicas de los profesionales de cuidados paliativos y atención psicosocial. Se refuerzan su voluntad y capacidad de adecuación a las

Capacidad de los profesionales para adaptarse a la situación nuevas necesidades acontecidas

- Han elaborado material didáctico e informativo, aunque no han llegado al público ideal y no ha sido consultado por los propios equipos de CP y EAPS

- Han respondido desde el inicio con fuerza y profesionalidad integrando también las emociones de miedo, tristeza, rabia, impotencia, etc., generadas por la situación. Emociones a las que va a ser necesario realizar un seguimiento y ofrecer soporte en caso de ser preciso

Capacidad de los pacientes y familias para adaptarse a la situación

- Tanto pacientes como familias han comprendido la gravedad extrema de esta situación y se han adaptado y aceptando los cambios y nuevos modelos de atención resultantes. Se cuestionan qué ocurrirá si la situación se prolonga o repite

- Se ha visto directamente afectado, sobre todo en un cambio de modalidad de atención que Adaptación del modelo de atención ha pasado, en la mayoría de casos, de ser presencial a telemática. Si bien este aspecto se valora como una acción necesaria y adaptativa para un primer momento, los expertos inciden que cabe replantear y analizar sus beneficios a largo plazo al considerase que pueda ser insuficiente en función del momento en que se encuentre la persona enferma

- Necesidad de revisar los modelos actuales de atención para adaptarlos, protocolizarlos y

Oportunidades y posible generación de cambios para la mejora de la calidad de la atención difundirlos de forma que pueda visualizarse y legitimarse la labor de los profesionales de cuidados paliativos y de la atención psicosocial en cualquier ámbito o territorio, alcanzando así una mayor cobertura y pudiendo de este modo ofrecer una atención integral, integrada y de calidad a todas las personas que lo necesiten, ya sean personas enfermas y/o sus familias y/o profesionales de otras disciplinas 


\section{Pasos adaptación fase postaguda}

Dentro de las respuestas obtenidas del grupo de expertos, se obtuvieron una serie de factores que se han plantean como relevantes a la hora de elaborar nuevas propuestas organizativas para la adaptación a la fase postaguda de la pandemia. Entre estos factores destacan (Figura 1):

1. Incertidumbre sobre la evolución temporal de la crisis y riesgo de nuevos brotes.

2. Aparición de nuevas necesidades:

Tipologías distintas de pacientes agudos, subagudos y crónicos.

- Atención paliativa provista por servicios diferentes (atención primaria, urgencias, agudos $\mathrm{UCl}$ y residencias).

- Aumento de necesidades de atención del duelo complicado.

- Aumento de necesidades de sobrecarga emocional en los profesionales sanitarios (fatiga por compasión, burnout, ansiedad, conciliación familiar, sobreidentificación con colegas afectados, por ejemplo).

3. Incremento de necesidades sociales, económicas, relacionales, entre otros, especialmente en personas mayores:

- Duelo y rehabilitación de los equipos y organizaciones. - Aumento de la necesidad de rehabilitación de las secuelas por COVID-19: salud mental, física.

- Consecuencias derivadas del retraso diagnóstico, la evolución o descompensación de patologías crónicas previas.

- Posible aparición de "clústeres" de necesidades asociadas a la enfermedad avanzada: asociación de due- lo + soledad + pobreza + aislamiento + enfermedad avanzada.

\section{Evaluación cualitativa de percepción de capacidad de adaptación al reto COVID-19}

Los resultados principales de las opiniones recogidas en el análisis DAFO los podemos observar en las Tablas III y IV.

\section{Requisitos adaptación y éxito: liderazgo, compromiso y actitudes como factores más determinantes}

Dentro de las respuestas aportadas por el grupo de expertos se expusieron algunas características que deberían tener o desarrollar los equipos para poder afrontar la nueva época pos-COVID-19. Entre ellas destacaban:

1. Estado del equipo y recursos, y autoevaluación de respuesta a la COVID-19.

2. Liderazgo, autoestima, actitud positiva, proactiva, propositiva y abierta.

3. Posición en sistema de expertos en atención paliativa, psicosocial y espiritual.

4. Flexibilidad, adaptación al cambio y propuestas innovadoras.

5. Visión de oportunidad versus amenaza.

6. Confianza, colaboración y solidaridad con otras disciplinas y equipos en la elaboración de respuestas.

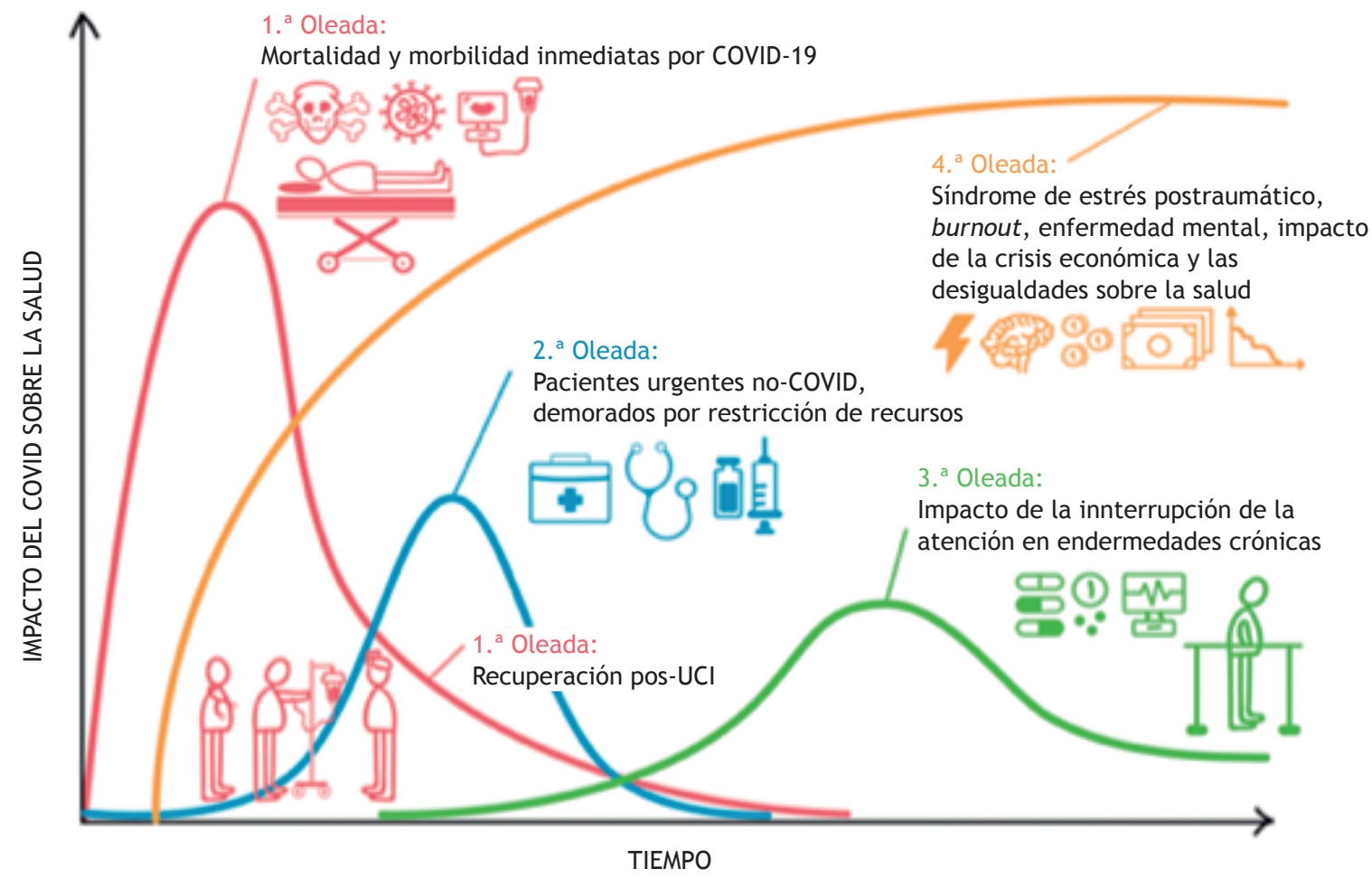

Figura 1. Modelo evolutivo de necesidades durante la pandemia por COVID-19 (Meneu y cols. ${ }^{27}$.) 
7. Aumentar visibilidad frente a los profesionales, gestores y medios de prensa.

8. Visión de sistema y comunidad.

9. Evaluación y análisis bioético de la respuesta.

10. Conocimiento de procesos de innovación y de implementación.

\section{Las acciones a implementar en la fase postaguda (Tabla II)}

En la Tabla II se recogen algunas de las propuestas recomendadas para implementar posfase aguda de la COVID-19 y para afrontar nuevas reagudizaciones de la pandemia. En la elaboración de propuestas se debe tener en cuenta:

1. Valoración de la capacidad de respuesta a la fase aguda y situación equipo (recursos, personas, etc.).

2. Análisis de las nuevas necesidades: tipología de pacientes, atención duelo, impacto profesionales.

3. Compartida con grupos de interés (servicios y equipos receptores, profesionales-clave).

4. Contacto con líderes sociales y programes comunitarios.

Para elaborar propuestas:

5. Sobre cómo preservar las funciones previas del equipo.

6. De nuevos targets de pacientes, familias y profesionales.

7. Nuevos escenarios: residencias, servicios urgencias y $\mathrm{UCl}$.

8. Nuevas fórmulas de intervención telemáticas, online, grupales, etc.

9. Interacción con programas sociales (comunidades cuidadoras, etc.).

10. De respuesta a nuevas crisis humanitarias o pandemias.

\section{Comentarios de los expertos}

Se ha realizado un análisis de contenido temático a partir de la transcripción literal y sistemática de la información obtenida en las encuestas abiertas autoadministradas realizadas a los expertos en cuidados paliativos y atención psicosocial, obteniendo comentarios específicos de 17 de los 26 expertos que han colaborado (Tabla V).

\section{DISCUSIÓN}

La crisis por la COVID-19 originó una respuesta inicialmente centrada en la protección al contagio y los servicios agudos hospitalarios, más tarde desplazada hacia la atención primaria, y en la que la atención paliativa apareció en una segunda fase de manera espontánea, no siempre liderada por los equipos específicos.

La respuesta a la crisis de la COVID-19 de los SCP y EAPS ha sido variable, desde el liderazgo activo de la atención paliativa a otras menos activas, o con uso mayoritario de la intervención telemática. Esta última posición ha sido mayoritaria en los EAP, y principalmente en los programas de final de vida y soledad y en los de comunidades compasivas (en todos aquellos que se basaban en la intervención presencial de voluntariado o de actos sociales). Constatamos que un grupo mayoritario de líderes consultados expresa quejas sobre el rol escaso de los SCP y EAP en la respuesta a la crisis, liderada por servicios de agudos inicialmente y de AP posteriormente, mientras que otro grupo en cambio, expresa una posición proactiva positiva.

Cabe destacar la importancia de la posición previa en el sistema, la capacidad de influencia y la relación previa con otros servicios como factores determinantes de la capacidad de reacción y la presencia en la crisis COVID-19, con rango amplio desde el liderazgo de la respuesta a la marginalidad, así como la flexibilidad, la adaptación y la capacidad de generar nuevas fórmulas de intervención y nuevas alianzas que respondan a nuevas necesidades y demandas como un factor clave para aprovechar y liderar las oportunidades que la crisis COVID-19 abre para la atención paliativa.

Se destaca la crisis por la COVID-19 como una experiencia que ha mostrado grandes necesidades no cubiertas de atención paliativa, con respuestas activas espontáneas individuales o colectivas, de profesionales o sociedad, así como necesidades emergentes a medio plazo como las del duelo complicado, las secuelas físicas y psicológicas de la pandemia y las sociales, con aumento de situaciones de vulnerabilidad. También ha mostrado necesidades en servicios como las residencias u otros de agudos, que abren nuevas perspectivas. Existe también incertidumbre sobre la evolución de la pandemia.

En general, ante estos retos de necesidades, se abren oportunidades de nuevas tipologías de pacientes o de servicios (residencias, urgencias y $\mathrm{UCl}$ ), de modelos de intervención (telemáticos), de desarrollo de áreas como la del duelo, el soporte de profesionales, y también de comunidades compasivas. Para responder activamente a estos retos, los SCP y EAPS requieren liderazgo, flexibilidad, adaptación y compromiso, y deben reordenar y adaptar los servicios en colaboración con los demás elementos del sistema.

\section{Limitaciones de este artículo}

La revisión de la literatura no ha sido sistemática por razones de premura, y la consulta a líderes seleccionados con criterios territoriales y de presencia en CP y EAPS ha sido elaborada y ejecutada también con urgencia. Con todo, refleja las distintas opciones de respuesta, y propone requisitos, criterios, y métodos para la adaptación posterior. Debe ser complementado con una revisión más sistemática y un estudio descriptivo que describan con mayor exactitud las respuestas y los retos de futuro.

\section{Impacto para la práctica y la definición de estrategias}

Este artículo parte de una propuesta compartida. Ha sido diseñado y realizado con criterios de participación y de respuesta rápida ante la crisis COVID. Sus limitaciones más relevantes provienen de la necesidad de una reflexión ante 
la crisis, que permitiera una descripción de las respuestas iniciales de los SCP y EAPS basadas en las experiencias nacionales y la literatura accesible (Tabla I), así como una propuesta metodológica (Tabla II) evolutiva sobre cómo elaborar medidas de adaptación a la nueva realidad pos-COVID-19. Estos hechos son considerados en conjunto como una oportunidad para los CP y la atención psicosocial.

Asimismo, de esta reflexión se proponen medidas específicas para cada servicio y elementos que deben tenerse en consideración para elaborar políticas de respuesta ante la crisis por la COVID-19 u otras situaciones de crisis humanitarias.

\section{*Listado de revisores externos (por orden alfabético)}

Alberto Alonso, Juan Pedro Arbizu, Jaime Boceta, Helena Camell, Carlos Centeno, Emma Costas, Teresa Donada, Julio Gómez Cañedo, Rafael Gómez García, Joaquim Limonero, Mercé Llagostera, Antonio Llergo, Paco López-Espinosa, Jorge Maté, Dolors Mateo, Teresa Mateu, Alberto Meléndez, Rafael Mota, Paco Vara, Sebastià Santaeugenia, y Lori Thompson.

Este trabajo ha contado con el soporte de Sociedad Española de Cuidados Paliativos (SECPAL) y Societat CatalanoBalear de Cures Pal-liatives.

\section{BIBLIOGRAFÍA}

1. Gómez-Batiste X, Amblàs J, Terribas N, Casellas-Grau A, Costa $X$, Román $B$, et al. Recomendaciones éticas y clínicas para la toma de decisiones en el entorno residencial en contexto de la crisis COVID-19 [Internet]. Infografía; 2020 [acceso el 16 de junio de 2020]. Disponible en: https://drive.google.com/file/ d/1i1ry0HPHD8zcl1kLS9L4GSGpTU3QO3KL/view?usp=sharing.

2. Amblàs-Novellas J, Gómez-Batiste X; en representación de los profesionales y organizaciones que han participado en el consenso. Clinical and ethical recommendations for decision-making in nursing homes in the context of the COVID-19 crisis. Med Clin (Barc). 2020;155:356-9.

3. Rubio O, Estella A, Cabre L, Saralegui-Reta I, Martin MC, Zapata $L$, et al. Recomendaciones éticas para la toma de decisiones difíciles en las unidades de cuidados intensivos ante la situación excepcional de crisis por la pandemia por COVID-19: revisión rápida y consenso de expertos. Med Intensiva. 2020;44:439-45.

4. Fundación "la Caixa": Programa para la atención integral a personas con enfermedad avanzada de la Obra Social "la Caixa" [Internet]. Barcelona: Observatorio "QUALY” UVIC/ICO; c2020. [citado el 15 de julio de 2020]. Directorio de recursos sobre COVID-19 para profesionales de la salud; [1 pantalla]. Disponible en: https: //obrasociallacaixa.org/es/atencion-integralenfermedades-avanzadas-la-caixa.

5. Fundación "la Caixa”: Línea de asistencia psicológica para profesionales sanitarios,. [Internet]. Barcelona: Fundación "la Caixa”. 2020 [citado el 15 de julio de 2020]. Cuidar a quienes nos cuidan. Disponible en: https://obrasociallacaixa.org/es/ cuidar-a-quienes-nos-cuidan.

6. Fundación "la Caixa”: Programa para la atención integral a personas con enfermedad avanzada de la Obra Social "la Caixa" [Internet]. Barcelona: Observatorio "QUALY" UVIC/ICO; 2020. [citado el 15 de julio de 2020]. Directorio de recursos sobre COVID-19 para profesionales de la salud. Disponible en: https: // obrasociallacaixa.org/es/atencion-integral-enfermedadesavanzadas-la-caixa.

7. Gómez-Batiste X. Atención paliativa COVID-19: prevenir, curar y cuidar. La Vanguardia (Barcelona). 23 de marzo de 2020. Disponible en: https: / / www.lavanguardia.com/opinion/20200323/ 4840637021 /atencion-paliativa-covid-19-prevenir-curar-y-cuidar.html.

8. Gómez-Batiste X, Calvo T, Gómez. J. Epidemiología del sufrimiento y práctica de la compasión. ABC-Salud (España). 18 de abril de 2020. Sección opinión. p. 26.

9. Downar J, Seccareccia D; Associated Medical Services Inc. Educational Fellows in Care at the End of Life. Palliating a pandemic: "all patients must be cared for". J Pain Symptom Manage. 2010;39(2):291-5.

10. Thompson AK, Faith K, Gibson JL, Upshur RE. Pandemic influenza preparedness: an ethical framework to guide decision-making. BMC Med Ethics. 2006;7:E12.

11. White DB, Katz MH, Luce JM, Lo B. Who should receive life support during a public health emergency? Using ethical principles to improve allocation decisions. Ann Intern Med. 2009;150:132-8.

12. World Health Organization. Integrating palliative care and symptom relief into responses to humanitarian emergencies and crises: a WHO guide. World Health Organization; 2018. Disponible en: https://apps.who.int/iris/handle/10665/274565.

13. Powell RA, Schwartz L, Nouvet E, Sutton B, Petrova M, Marston $J$, et al. Palliative care in humanitarian crises: always something to offer. Lancet. 2017;389(10078):1498-9.

14. Costantini M, Sleeman KE, Peruselli C, Higginson IJ. Response and role of palliative care during the COVID-19 pandemic: A national telephone survey of hospices in Italy. Palliat Med. 2020;34:889-95.

15. Etkind SN, Bone AE, Lovell N, Cripps RL, Harding R, Higginson IJ, et al. The Role and Response of Palliative Care and Hospice Services in Epidemics and Pandemics: A Rapid Review to Inform Practice During the COVID-19 Pandemic. J Pain Symptom Manage. 2020;60:e31-e40.

16. Rosenbaum L. Facing Covid-19 in Italy - Ethics, Logistics, and Therapeutics on the Epidemic's Front Line. N Engl J Med. 2020;382:1873-5.

17. Powell VD, Silveira MJ. What Should Palliative Care's Response Be to the COVID-19 Pandemic? J Pain Symptom Manage. 2020;60:e1-e3.

18. Wallace CL, Wladkowski SP, Gibson A, White P. Grief During the COVID-19 Pandemic: Considerations for Palliative Care Providers. J Pain Symptom Manage. 2020;60:e70-e76.

19. Wakam GK, Montgomery JR, Biesterveld BE, Brown CS. Not Dying Alone - Modern Compassionate Care in the Covid-19 Pandemic. N Engl J Med. 2020;382:e88.

20. International Association for Hospice and Palliative Care: Palliative care and COVID-19. [Internet]; Houston: IAHPC. c2020. [citado el 15 de julio de 2020]. Disponible en: http: / / globalpalliativecare.org/covid-19.

21. Arya A, Buchman S, Gagnon B, Downar J. Pandemic palliative care: beyond ventilators and saving lives. CMAJ. 2020;192(15): E400-E404.

22. Bowman BA, Back AL, Esch AE, Marshall N. Crisis Symptom Management and Patient Communication Protocols Are Important Tools for All Clinicians Responding to COVID-19. J Pain Symptom Manage. 2020;60:e98-e100.

23. Bajwah S, Wilcock A, Towers R, Costantini M, Bausewein C, Simon ST, et al. Managing the supportive care needs of those affected by COVID-19. Eur Respir J. 2020;55(4):2000815.

24. Chidiac C, Feuer D, Naismith J, Flatley M, Preston N. Emergency Palliative Care Planning and Support in a COVID-19 Pandemic. J Palliat Med. 2020;23:752-3.

25. Humphreys J, Schoenherr L, Elia G, Saks NT, Brown C, Barbour $S$, et al. Rapid Implementation of Inpatient Telepalliative Me- 
dicine Consultations During COVID-19 Pandemic. J Pain Symptom Manage. 2020;60:e54-e59.

26. Fundación "la Caixa": Programa para la atención integral a personas con enfermedad avanzada de la Obra Social "la Caixa" [Internet]. Barcelona: Observatorio "QUALY" UVIC/ICO; 2020. [citado el 15 de julio de 2020]. Directorio de recursos sobre COVID-19 para profesionales de la salud. Disponible en: https:// obrasociallacaixa.org/es/atencion-integral-enfermedadesavanzadas-la-caixa.

27. Meneu R, Ortun V, Peiró, González B, Hernández I, Ibern P. La salida: Mejorar la capacidad de respuesta sanitaria (y social). Blog Economía y Salud, AES. 19 abril 2020. Disponible en: http: / / www. aes.es/blog/2020/04/19/la-salida-mejorar-lacapacidad-de-respuesta-sanitaria-y-social/. 